\title{
Factors Influencing Speech and Swallowing Outcomes after Oral Resections
}

\author{
Dr Anitha Gandhi $\mathrm{Mch}^{1}$,Dr Sivakumari Sivarajan $\mathrm{Mch}^{2}{ }^{2} \mathrm{Mrs}$ S.Jeya ${ }^{3}$ \\ ${ }^{I}$ (Department of Surgical Oncology, Tirunelveli Medical College,Tamilnadu, India) \\ ${ }_{2}^{2}$ (Department of Surgical Oncology Govt. Omanthurar Multisuperspeciality Hospital, Tamilnadu, India ) \\ 3 ( Speech Language Pathologist, Department Of Ent, Madras Medical College, Tamilnadu, India)
}

\begin{abstract}
Surgery of oral cavity cancers is often extensive requiring complex reconstructive procedures which affect functions of speech and swallowing.The aim of the present study was to assess speech and swallowing outcome after various surgeries for oral cavity cancers in an objective manner.This is a retrospective study of 31 patients with oral cavity cancers who underwent surgery in our hospital. Swallowing assessment was performed by video fluoroscopy using barium. Speech was assessed objectively for both articulation and intelligibility. The average interval between surgery and evaluation was 8 months. Fifty percent of patients had preoperative chemoradiation (10), radiation (3) or chemotherapy (2). The swallowing was normal in $51 \%$ of patients whereas the remaining patients had swallowing problems. Speech assessment scores were $>75 \%$ in $26 \%$ of patients, $74 \%$ of patients had scores in the range of 51-75\%. Preop RT affected both speech and swallowing outcome significantly. Mandibular resection significantly worsened the swallowing $(p=0.001)$ and speech (0.014). The extent of soft tissue resection and type of reconstruction also made significant impact. Functional outcome of speech and swallowing are mainly influenced by subsite involved, extent of bone and soft tissue resection, type of reconstruction and radiotherapy
\end{abstract}

Keywords: dysphagia, functional outcomes, oral resections, speech, swallowing,

\section{Introduction}

Oral cavity is a functionally important area essential for speech and swallowing. Surgical resections for oral cancers are often extensive involving complex reconstructive procedures leading to impairment of speech and swallowing . Despite improvements in the reconstructive armamentarium including microvascular free flaps and sensate flaps the magnitude of these functional impairments still persists which in turn adversely affect the psychosocial wellbeing of the patients.

\section{Aim}

The aim was to assess speech and swallowing outcomes and factors influencing them after oral cancer resections .

\section{Patients and methods}

This is a retrospective study of 31 patients with oral cavity squamous cell carcinoma who underwent surgery in a tertiary care cancer centre from February 2011 to June 2012. Swallowing was assessed by subjecting the patient to videofluoroscopic examination and the images were recorded both in anteroposterior and lateral views in a videotape.The material used was barium either in paste or liquid form whichever the patient was able to consume. The recordings were observed for oropharyngeal delay, retention of barium in vallecula, penetration and aspiration and were scored as $0:$ normal swallowing, 1: retention of barium in vallecula, 3 : penetration, $4:$ aspiration for ease of statistical analysis. Penetration is defined as any portion of the bolus entering the laryngeal vestibule to the level of (but not passing below) the vocal folds. Aspiration occurs once the penetrated material passes the level of the vocal folds and enters the subglottic region[1]. Speech evaluation was done by speech pathologist for both articulation and intelligibility. Standard articulation tests were used for assessment where the patient is asked to read and was observed for substitution, addition, distortion, omission errors. Intelligibility was assessed from patient's spontaneous speech. Combining these two factors speech was scored as $<50 \%, 51-75 \%,>75 \%$ with $>75 \%$ being the best score.

The statistical analysis was done with SPSS software version 16.0

\section{Results}

31 Patients were included in the study. $77 \%$ of the patients were males $(n=24)$ and $23 \%$ were females $(\mathrm{n}=7)$. Mean age was 48 years (range: $26-65 \mathrm{yrs})$. The commonest subsite involved in our series was tongue $(n=13)$ followed by buccal mucosa $(n=11)$, floor of mouth $(n=3)$, retromolar trigone $(n=2)$, upper and lower alveolus ( $\mathrm{n}=1$ each). Mean follow up period at the time of evaluation was 8 months. $50 \%$ of the patients had pre- 
operative treatment either in the form of chemoRT $(n=10), R T(n=3)$, chemo $(n=2)$. The T stage of the patients were as follows : T1 in $11, \mathrm{~T} 2$ in 10, T3 in 2, T4 in 8 patients. Nodal staging was N0 in 19 and N+ in 12 patients. The type of resections included wide local excision, hemiglossectomy, palatoalveolar resction, composite resection. The reconstruction techniques used were pedicled flaps, including nasolaial flap, tongue flap, Pectoralis major myocutaneous flap and deltopectoral flap, SSG, and prosthetic reconstruction with obturator. Swallowing was found to be normal in 10 out of 11 patients $(90 \%)$ who were treated with wide local excision alone and did not require any reconstruction. Among the patients who had reconstruction $(\mathrm{n}=20), 70 \%$ had swallowing problems in the form of retention of barium $(n=12)$ and penetration $(n=2)$. Only $30 \%(n=6)$ patients had normal swallowing. Within the reconstructed group of patients, those who had their reconstruction in the form of SSG $(n=2)$, obturator $(n=1)$, tongue flap $(n=2)$ had normal swallowing whereas $90 \%(n=14)$ of patients with pedicled flap reconstruction $(n=15)$ had swallowing abnormality. The speech scores were $>75 \%$ in one third of the patients $(26 \%)$ and the remaining two thirds had an intermediate score of 51-75\%. None had a score $<50 \%$. The factors found to impact on speech and swallowing outcomes were the subsite involved where tongue lesions produced worse outcome of both speech and swallowing followed by floor of mouth $(\mathrm{p}=0.114)$ and among the treatment modalities, radiotherapy was associated with reduced scores of both $\operatorname{speech}(\mathrm{p}=0.556)$ and swallowing $(\mathrm{p}=0.016)$. The more the extent of resection the worse is the outcome for both speech $(\mathrm{p}=0.003)$, and swallowing $(\mathrm{p}=<0.01)$. With respect to reconstructive techniques, less demanding procedures ( primary closure, SSG) had best functional outcome on both speech $(\mathrm{p}=0.007)$ and swallowing $(\mathrm{p}=0.005)$ than flap reconstructions .

\section{Discussion}

Assessment of oral functions after radical surgery is complex due to involvement of numerous interaction variables [2]. Most of the studies done on this aspect are mainly subjective where the outcomes can be either overestimated or underestimated. Here comes the importance of objective assessment which will reveal the actual magnitude of problem. Such studies are limited in number and the current study is one among these where the speech and swallowing outcomes assessment were done in objective manner. The results of the present study clearly demonstrate the problem of speech and swallowing impairment following treatment in oral cancers. Factors adversely affecting swallowing outcome included preoperative treatment with radiotherapy, subsite involved particularly tongue and floor of the mouth, extensive soft tissue resection, bone resection, type of reconstruction (pedicled flaps). Whereas impact on speech was by subsite involved, extent of soft tissue and bone resection and type of reconstruction. The results of the present study correlate well with the other similar studies reported in literature.

\subsection{Comparison with other studies}

Studies by Schliephake et al, Mc connel et al Samir S Khariwala et al revealed thenegative impact of tongue resections in speech and swallowing. This is because tongue is the dynamic structure involved in propulsion of food into pharynx . oral tongue resection is associated with prolonged oral preparatory time [14], slowed oral transit time [14,15], increased oral residue [15], and increased pharyngeal residue [15].Tongue base resections of $>25 \%$ predisposes to severe post surgical aspiration due to inability to clear bolus from pharynx. Not only the tongue base, but also the oral tongue resection can produce negative impact on swallowing function. Maria mercedez surez et al [2] found that floor of mouth resections too contributed to swallowing impairment. This is probably due to associated removal of geniohyoid and mylohyoid leading to impaired hyolaryngeal elevation and ultimately retention of food in pyriform sinus and aspiration [17].

Even buccal mucosa resection contributes to dysphagia since it is important for oral preparatory phase and for preventing food spillage into lateral sulci [18]. This negative impact of resection of oral cavity subsites is further confirmed by Hara et al 's study on his cohort of 25 patients [19].

In the present study neck dissection done during composite resection also contributed to dysphagia which reveals statistical significance $(\mathrm{p}=0.000)$. This is supported by the fact that denervation of strap muscles due to sacrifice of ansa cervicalis can cause dysphagia.In our study, preoperative RT was the most significant among factors associated with worse swallowing and speech outcomes. This finding is confirmed in studies by various authors including Zuydam et al, Matsui et al. This negative effect of RT is mainly due to inclusion of critical anatomical structures in head and neck region in the radiation field and the resulting fibrosis. The fibrosis in turn leads to impaird mobility of tongue, reduced tongue base retraction, residue in vallecula and pyriform sinus, reduced laryngeal elevation.[16] Apart from these direct effects, additional factors contributing to swallowing dysfunction include xerostomia, hypopharyngeal and oesophageal strictures(8-24\%) .

The more the extent of resection the more was the functional impairment. This observation made in our study correlates with the studies by Zuydam AC et al [3] and Anna Karinne [5] et al. Regarding the impact of bone resection, marginal mandibulectomy did not influence the outcomes since ther is no disruption of mandibular arch. In situations where hemimandibulectomy is performed as a part of composite resection, the 
remaining functional segment often deviates to surgical side. In addition removal of mandible obliterate portions of lingual and buccal sulci so that proper channelling of secretions is affected. Hemimandibulectomy is a negative prognosticator for both speech $(\mathrm{p}=0.014)$ and swallowing $(\mathrm{p}=0.000)$ in the present study. Schilephake $\mathrm{V}$ et al observed in his study on evaluation of quality of life mandibular resection was associated with reduced scores [21].

Among the various reconstructive techniques, patients with their defects closed primarily fared better than those who were reconstructed with either pedicled / free flaps. Mc connel et al, Suerez et al found similar results. This in turn may be due to lesser extent of resection which do not necessitate any reconstruction (eg. Hemiglossectomy). This finding stresses the importance of mobility of the remaining tongue[11] for speech and swallowing which is compromised in pedicled flap reconstruction where flap is sutured to the remaining tongue. Moreover these flaps are bulky and adynamic which interferes with the mobility of remaining normal oral tissues thereby reducing the swallowing efficiency. Although these problems are overcome with free flaps and there are studies to confirm this, some comparative studies reveal no difference between the two [10,12].

Even with the use of sensate flaps which retain their innervation in addition to blood supply, there still exists the problem of swallowing impairment. Though Markkanen-Leppanen et al, Yu P et reported superior results with sensate flaps [19,20], Mah SM [21],found no significant difference between sensate and non sensate flaps.

The prevalence of speech and swallowing problems observed in the present study is comparable with other studies and is shown in Table 1

Table 1

\begin{tabular}{|l|l|l|l|l|l|l|}
\hline & $\begin{array}{l}\text { Number of } \\
\text { patients }\end{array}$ & $\begin{array}{l}\text { Type of } \\
\text { reconstruction }\end{array}$ & $\begin{array}{l}\text { Retention of } \\
\text { barium } \\
\text { vallecula }\end{array}$ & $\begin{array}{l}\text { penetration } \\
\text { in }\end{array}$ & aspiration & $\begin{array}{l}\text { Speech } \\
\text { scores }\end{array}$ \\
\hline Our study & 31 & $\begin{array}{l}\text { Pedicled flaps and } \\
\text { primary closure }\end{array}$ & $42 \%$ & $6 \%$ & $0 \%$ & $76 \%$ \\
\hline $\begin{array}{l}\text { Archontaki et al } \\
{[22]}\end{array}$ & 20 & Free flap & $25 \%$ & $10 \%$ & $15 \%$ & $68 \%$ \\
\hline Borggreven et al & 80 & Free flap & - & $38-47 \%$ & $34-25 \%$ & - \\
\hline $\begin{array}{l}\text { Markkanen- } \\
\text { Leppanen et al }\end{array}$ & 41 & Free flap & $48 \%$ & - & $36 \%$ & $84 \%$ \\
\hline
\end{tabular}

\section{Conclusion}

The present study confirms the facts on the existing literature devoted to the topic of postoperative functional outcomes of speech and swallowing in head and neck cancers. Speech and swallowing functions were affected by subsite involved, extent of bone and soft tissue resection, type of reconstruction and radiotherapy. The information obtained from the present study provides better insight into factors predicting postoperative outcomes. This in turn will be helpful for better pretreatment patient counselling, proper intra operative planning regarding resection and reconstruction and instituting early post operative rehabilitation.

Nil

\section{Acknnowledgements}

\section{Conflict of Interest : Nil}

\section{Rererences}

[1]. Objective Assessment of Swallowing Function After Definitive Concurrent (Chemo)radiotherapy in Patients with Head and Neck Cancer Jaiprakash Agarwal • Vijay Palwe • Debnarayan Dutta・Tejpal Gupta •Dysphagia (2011) 26:399 406

[2]. Speech and Swallowing Impairment After Treatment for Oral and Oropharyngeal Cancer FREEMaria-Mercedes Suarez-Cunqueiro, DDS, PhD; Alexander Schramm, DDS, MD, PhD; Ralf Schoen, DDS, MD,PhD Arch Otolaryngol Head Neck Surg. 2008;134(12):1299-1304. doi:10.1001/archotol.134.12.129

[3]. Predictors of speech and swallowing function following primary surgery for oral and oropharyngeal cancer Zuydam, A.C., ${ }^{*}$ Lowe, D. † Brown, J.S., † Vaughan, E.D., \& \& Rogers, S.N., Clin. Otolaryngol. 2005, 30, 428-437

[4]. Swallowing Outcomes After Microvascular Head and Neck Reconstruction: A Prospective Review of 191 Cases Samir S. Khariwala, MD; Prashant P. Vivek, MD; Robert R. Lorenz, MD; Ramon M. Esclamado, MD; Laryngoscope, 117:1359-1363, 2007

[5]. Analysis of swallowing afterretromolar or oropharynx resection and reconstruction with myocutaneous or microvascular free flaps Anna Karinne C. Bandeira ${ }^{1}$, Maria de Fátima G.G. Tomazelli ${ }^{2}$,Luciana P. do Vale-Prodomo ${ }^{1}$ Applied Cancer ResearchFAQ Vol 27, No 1(2007).

[6]. Functional Results of Primary Closure vs Flaps in Oropharyngeal Reconstruction A Prospective Study of Speech and Swallowing Fred M. S. McConnel, MD; Barbara Roa Pauloski, PhD; Jerilyn A. Logemann, PhD Arch Otolaryngol Head Neck Surg. 1998;124(6):625 630.

[7]. Swallowing after major surgery of the oral cavity or oropharynx: A prospective and longitudinal assessment of patients treated by microvascular soft tissue reconstruction Pepijn A. Borggreven $\mathrm{MD}^{1}$, Irma Verdonck-de Leeuw SLP, PhD ${ }^{1, *}, \mathrm{Rico}^{\mathrm{N}}$. Rinkel MD ${ }^{1}$ Head \& Neck Volume 29,_Issue 7,_pages 638-647, July 2007.

[8]. Speech, deglutition and life quality after intraoral tumour resection. Schliephake H, Schmelzeisen R, Schonweiler R. et al. (1998 A prospective study. Int. J. Oral Maxillofac. Surg. 27, 99-105. 
[9]. Nicoletti G, Soutar DS, Jackson MS, Wrench AA, Robertson G. Chewing and swallowing after surgical treatment for oral cancer: functional evaluation in 196 selected cases. Plast Reconstr Surg 2004;114 (2) 329- 33.

[10]. McConnel FMS, Teichgraeber JF, Alder RK. A comparison of three methods of oral reconstruction. Arch Otolaryngol Head Neck Surg. 1987;113496- 500

[11]. Su WF, Hsia YJ, Chang YC, Chen SG, Sheng H (2003) Functional comparison after reconstruction with a radial forearm free Xap or a pectoralis major Xap for cancer of the tongue. Otolaryngol Head Neck Surg 128(3):412-418.

[12]. 14.Pauloski BR, Logemann JA, Rademaker AW, et al. Speech and swallowing function after anterior tongue and floor of mouth resection with distal flap reconstruction. J Speech Hear Res 1993;36:267-276. [PubMed: 8487519]

[13]. Furia CL, Carrara-de Angelis E, Martins NM, et al. Videofluoroscopic evaluation after glossectomy. Arch Otolaryngol Head Neck Surg 2000;126:378-383. [PubMed: 10722012]

[14]. Rehabilitation of Dysphagia Following Head and Neck Cancer.Barbara R. Pauloski, PhDa Phys Med Rehabil Clin N Am. 2008 November ; 19(4): 889-928.

[15]. Hirano M, Kuroiwa Y, Tanaka S, et al. Dysphagia following various degrees of surgical resection for oral cancer. Ann Otol Rhinol Laryngol 1992;101:138-141

[16]. Speech and Swallowing Outcomes in Buccal Mucosa Carcinoma. Sunila John, Rashida M Hassuji, and B Rajashekhar Indian J Palliat Care. 2011 Sep-Dec; 17(3): 238-240

[17]. Hara I, Gellrich NC, Duker J, et al. Swallowing and speech function after intraoral soft tissue reconstruction with lateral upper arm free flap and radial forearm free flap. Br J Oral Maxillofac Surg 2003;41:161-169.

[18]. Markkanen-Leppanen M, Isotalo E, Makitie AA, et al. Swallowing after free-flap reconstruction in patients with oral and pharyngeal cancer. Oral Oncol 2006;42:501-509.

[19]. Yu P. Reinnervated anterolateral thigh flap for tongue reconstruction. Head Neck 2004;26:1038-1044.

[20]. Mah SM, Durham JS, Anderson DW, et al. Functional results in oral cavity reconstruction using reinnervated versus nonreinnervated free fasciocutaneous grafts. J Otolaryngol 1996;25:75-81.

[21]. Long-term quality of life after ablative intraoral tumour surgery. Schliephake H, Neukam FW, Schmelzeisen R, Varoga B, Schneller H. J Craniomaxillofac Surg. 1995 Aug;23(4):243-9.

[22]. Maria Archontaki, Athanasios Athanasiou, Spyros D.Stavrianos. Functional results of speech and swallowing after oral microvascular free flap reconstruction. Eur Arch Otorhinolaryngology (2010) 267: 1771-1777 